SICLOPPS cyclic peptide libraries in drug discovery.

Ali Tavassoli ${ }^{\mathrm{a}}$

${ }^{a}$ Chemistry, University of Southampton, Southampton, SO17 1BJ, United Kingdom. e-mail:

ali1@soton.ac.uk

Cyclic peptide libraries have demonstrated significant potential when employed against challenging targets such as protein-protein interactions. While a variety of methods for library generation exist, genetically encoded libraries hold several advantages over their chemically synthesized counterparts; they are more readily accessible and allow straightforward hit deconvolution. One method for the intracellular generation of such libraries is split-intein circular ligation of peptides and proteins (SICLOPPS). Here we detail and discuss the deployment of SICLOPPS libraries for the identification of cyclic peptide inhibitors of a variety of targets.

\title{
Highlights
}

- SICLOPPS allows the rapid generation of cyclic peptide libraries in cells.

- A variety of cyclic peptide ring sizes may be generated with SICLOPPS.

- SICLOPPS libraries contain up to $\sim 10^{9}$ unique members.

- SICLOPPS can be combined with any cell-based assay for inhibitor identification.

- SICLOPPS has been employed against a multitude of 'difficult' targets in various organisms.

\section{Introduction to SICLOPPS}

The use of cyclic peptides in drug discovery has come of age, with several successful companies established on the back of genetically-encoded library generation technologies developed in academia [1-5]. These technologies allow rapid deconvolution of hits that bind to a target protein by sequencing the DNA or mRNA tag bound to (or compartmentalized in phage) with each peptide [6]. In a wider sense, the mRNA-display and phage-display technologies at the core of these methods are relatively similar in scope; the cyclic peptide libraries generated are screened in vitro using affinity-based assays. While synthetic cyclic peptide (or macrocycle) libraries may be generated and screened in functional assays [7,8], generating and screening libraries of comparable size to genetically-encoded ones $\left(\sim 10^{9}\right.$ members) requires significant effort and a high degree of automation.

An alternative, complementary method for cyclic peptide library generation is termed split-intein circular ligation of peptides and proteins (SICLOPPS) and takes advantage of intein splicing for cyclic peptide library generation in cells (Figure 1A). Inteins are self-excising protein domains that process to link their flanking sequences by a native peptide bond [9]. The SICLOPPS construct encodes a Cterminal intein domain followed by the sequence to be cyclized, and an $\mathrm{N}$-terminal intein domain. Upon transcription and translation, this gives an active cis-intein that cyclizes the polypeptide sequence 
between the $\mathrm{C}$-and $\mathrm{N}$-terminal intein domains as a byproduct of splicing (Figure 1B) [10]. And while SICLOPPS originally used the DnaE split-inteins from Synechocystis sp. PCC6803 (Ssp) [11], we have recently reported adaptation of the faster splicing engineered inteins from Nostoc punctiforme ( $\mathrm{Npu}$ ) for SICLOPPS [12]. Npu inteins are also significantly more tolerant of amino acid variations in the extein sequence, especially near the splice junctions, meaning that a larger proportion of a SICLOPPS library is cyclized by these inteins than $S s p$ inteins.

SICLOPPS is a simple and accessible method for cyclic peptide library generation; all that is required for the preparation of hundreds of millions of cyclic peptides is the SICLOPPS plasmid, a degenerate oligonucleotide, and a handful of straightforward molecular biology steps $[13,14]$. The degenerate oligonucleotide encodes the variable segment as repeats of NNS or NNB, where N represents any of the four DNA bases (A, C, G or T), S represents C or G; and B represents C, G or T. The NNS and NNB sequences cover 32 and 48 codons respectively and all 20 amino acids, while eliminating the ochre (UAA) and opal (UGA) stop codons from the library. For splicing to occur, the first amino acid of the target peptide needs to be a nucleophilic cysteine or serine, but there are no other limits on the number or identity of amino acids in the target peptide, allowing cyclic peptides of various sizes and sequences to be assembled. The number of randomized amino acids, as well as preset amino acids in a given position (e.g. a proline in position 3) are all controlled at the DNA level, and set during the design and synthesis of the degenerate oligonucleotide. Typically, 5 or 6 variable amino acids are introduced, as the total number of cyclic peptide library members $\left(3.2 \times 10^{6}\right.$ and $6.4 \times 10^{7}$ respectively $)$ will be lower than the maximum number of Escherichia coli (E. coli) transformants (typically $\sim 10^{9}$ ) ensuring that every member of the library is assayed. Although larger cyclic peptide rings containing more randomized amino acid positions can be also generated and screened, the coverage of such a library would be limited to the maximum transformation efficiency of the host. This plasmid library may be interfaced with any cell-based assay and screened for members of the library that confer the desired phenotype. Here, the use of SICLOPPS libraries in drug discovery is reviewed and discussed.

A

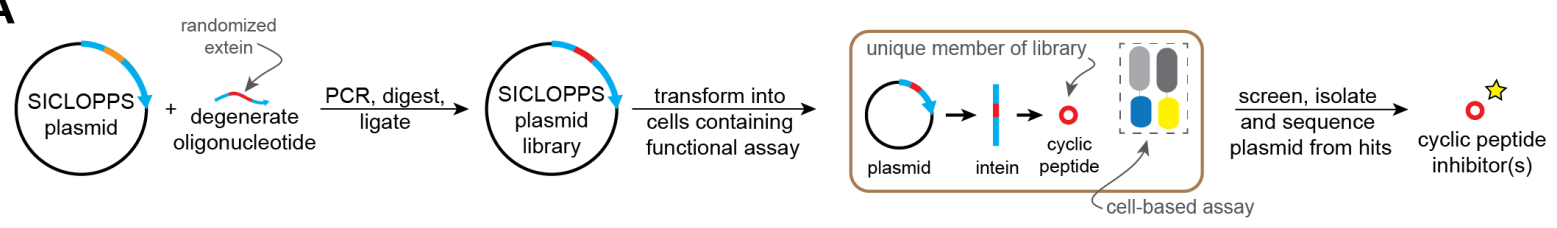

B

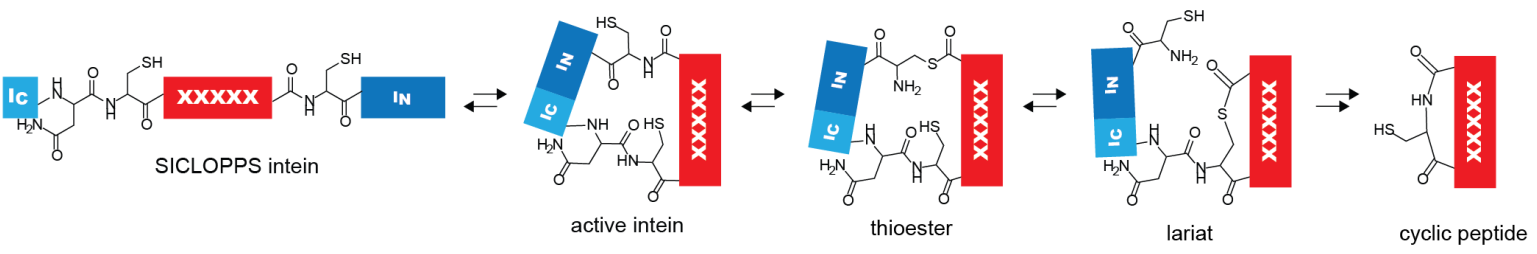

Figure 1. SICLOPPS library screening. A) Representative workflow for generating and screening a

SICLOPPS library. The library is generated using standard molecular biology techniques with a degenerate 
oligonucleotide which has been designed to determine the ring size of the cyclic peptides, number of randomized amino acids and any set amino acids. The plasmid library can then be transformed into cells containing an assay (e.g. FRET, life/death, phenotypic) and screened. The identity of the active cyclic peptides is revealed by isolating the SICLOPPS plasmids form cells hat show the desired phenotype, followed by DNA sequencing. B) The mechanism of SICLOPPS intein splicing. The two intein fragments, $\mathrm{I}_{\mathrm{N}}$ and $\mathrm{I}_{\mathrm{C}}$, interact to form an active intein that splices to cyclize the extein placed in between. In this case, a cyclic hexa-peptide library containing cysteine (required for intein splicing), followed by 5 random amino acids $(\mathrm{X}=$ any amino acid) is produced.

\section{The advantages and disadvantages of SICLOPPS}

The number of different academic groups that have successfully used SICLOPPS [15] attests to a key advantage of this system; namely, the simplicity, speed and ease with which libraries containing hundreds of millions of cyclic peptides can be created $[13,14]$. Another benefit is the ability to interface these libraries with assays in a variety of organisms; SICLOPPS inteins have been demonstrated to function in E. coli [16,17], yeast [18], and human cells [19,20]. SICLOPPS libraries are DNA-encoded, which gives a large amount of control over the makeup of the library and allows a variety of libraries to be easily produced and screened against a given target. Examples of variations in SICLOPPS libraries that are easy to implement include: cyclic peptides of different ring sizes, libraries with different amino acid composition (using limited codon sets), or inclusion of a given amino acid, or motif in a set position in every member of the library. Thus, the user has absolute control over the makeup of their cyclic peptide library via the degenerate oligonucleotide that encodes it.

Another advantage of SICLOPPS is that the cyclic peptide libraries are generated in cells, which allows functional assays to be conducted against a variety of targets, thus not only assessing affinity of each member of the library, but also its function against the given target (Figure 1A). It may be reasonably argued that functional screening is superior to affinity based screens (necessary with genetically-encoded cyclic peptide libraries generated in vitro) for inhibitor identification, as although affinity-based selection usually gives compounds that bind their target with very high-affinity, this does not equate to function, especially in cells.

A weakness of SICLOPPS libraries is that they are limited to using the 20 canonical amino acids as building blocks. Although SICLOPPS has been combined with an orthogonal aminoacyl-tRNA synthetase/tRNA $\mathrm{CUA}_{\mathrm{C} A}$ pair, to allow the incorporation of a single additional unnatural amino acid into SICLOPPS cyclic peptide libraries [21], mRNA-display methods have the potential to encode several non-natural amino acids at once via a custom in vitro transcription/translation (IVTT) system that uses reassigned tRNA charged with non-natural amino acids [22,23].

Another disadvantage of SICLOPPS libraries has been the relatively slow splice rate of the Ssp intens and their sensitivity to amino acid changes near the splice junctions [24], meaning that a significant 
portion of a cyclic peptide library may not actually be cyclic peptides, but rather exist as the partially spliced intein. However, these limitations have recently been overcome with the adaptation of the faster splicing and more promiscuous $\mathrm{Npu}$ inteins for SICLOPPS [12].

Another point of note is that the affinity of the hits isolated from SICLOPPS libraries tend to be in the high nanomolar to low micromolar region, which is weaker than the affinity of reported cyclic peptides identified using display-based libraries (typically low nanomolar). This difference is either due to the larger cyclic peptide rings (typically 14-mers or larger) generated in mRNA- and phagedisplay versus the 6-mers typically generated with SICLOPPS, or an artefact of using cell-based functional assays with SICLOPPS versus affinity based in vitro assays used with display-based libraries (that inherently find the tightest binding molecule). The answer is likely a combination of both these points. The tendency to generate libraries containing larger ring sizes in mRNA display is a consequence of the higher bandwidth of this method, which occurs in vitro and so is not limited by the transformation efficiency of the host. Thus, libraries as large as $10^{15}$ members may be generated and screened. However, it should be noted that the size of a 14-mer library randomized to allow all 20 canonical amino acids in each position is $8.3 \times 10^{22}$, therefore unless limited codon sets are used to significantly reduce the number of amino acids possible in each position, only $0.00001 \%$ of the library will be assessed in such a screen. It is likely that full coverage of a library is not critical for hit identification, and the number of random positions may be reduced through several rounds of screening and identifying set motifs. Regardless of this, larger peptides will have more contact with their target protein and so will have higher affinity for their target, and while nothing precludes cyclic peptides of similar ring size being generated by SICLOPPS (and a ratio of the library being assessed in cells), we have always reasoned that smaller cyclic peptides are more likely to form a single continuous point of contact with the target protein, and so will be easier to translate into drug-like small molecules than larger rings with multiple contact points, especially when targeting intracellular proteins. However, SICLOPPS libraries containing larger cyclic peptides should be generated and screened to probe the hypothesis that the difference in affinity with mRNA display derived hits is due to the smaller ring sizes used with SICLOPPS.

\section{SICLOPPS in prokaryotes}

SICLOPPS libraries can potentially be interfaced with any cell based assay in any organism [15]. In a frequently used iteration, SICLOPPS has been combined with an E. coli-based reverse two-hybrid system (RTHS) for the identification of cyclic peptide inhibitors of a variety of protein-protein interactions [16,17,25-27]. An advantage of this approach is that protein-protein interactions from other organisms may be incorporated into the E. coli RTHS, thus eliminating the possibility of off-target inhibitors, as the upstream and downstream protein partners will not be present in E. coli. This approach overcomes one of the main weaknesses of cell-based functional screening: that an identified 'inhibitor' may be giving the expected phenotype by acting upstream or downstream of the targeted proteins, or 
via a related signaling pathway. For example, human signaling cascades are not present in E. coli, eliminating the possibility of off-target inhibitors being identified. There are several reported examples of this, including identification of inhibitors of mammalian protein-protein interactions $[25,26,28]$. Of note is the hypoxia-inducible factor-1 (HIF-1) inhibitor identified with this system; cyclo-CLLFVY, the first isoform-specific inhibitor of the HIF- $1 \alpha / \mathrm{HIF}-1 \beta$ protein-protein interaction that acts by binding to the PAS-B domain of HIF-1 $\alpha$ with an affinity of $124 \mathrm{nM}$ [26]. Another example is the development of an E. coli RTHS for the interaction of the Staphylococcus aureus $\beta$-sliding clamp and its use to screen a SICLOPPS library; this identified cyclic peptides that inhibit dimerization of the $\beta$-sliding clamp, and therefore prevent DNA replication and lead to $S$. aureus cell death [29].

SICLOPPS libraries have also been used with other assays in E. coli, including a transposase-based screen for the identification DAM methyltransferase inhibitors [30], as well as a ClpXP protease activity assay that used inhibition of degradation of GFP fused to a ClpXP tag as the readout [31]. More recently, bacterial virulence factors have been targeted by combining SICLOPPS with E. coli-based assays for the activity of proteins important to this process. In the first example, the ability of the RNA chaperone Hfq to bind small RNA has been targeted using a fluorescence-based assay that linked expression of yellow fluorescent protein to inhibition of Hfq function. A SICLOPPS library was generated and screened in this engineered $E$. coli strain, leading to the identification of a cyclic peptide inhibitor of this regulatory protein used for virulence in pathogenic bacteria [32]. The same group have reported cyclic peptide inhibitors of gram negative $\sigma^{\mathrm{E}}$ pathway, which is critical for maintenance of cell envelope homeostasis by controlling the integrity and composition of the bacterial outer membrane. A fluorescence-based assay for the function of $\sigma^{\mathrm{E}}$ was established in E. coli and combined with SICLOPPS for the identification of cyclic peptides that bind this protein and inhibit $\sigma^{\mathrm{E}}$-dependent transcription [33].

These examples show the potential for screening SICLOPPS libraries in E. coli-based assays. Our RTHS platform is at the plug-and-play stage, with a multitude of published and unpublished proteinprotein interactions from different organisms having been targeted with this system in combination with SICLOPPS libraries. An area with significant potential is targeting bacterial virulence factors with SICLOPPS libraries, either by porting appropriate assays into non-pathogenic bacteria, or assaying directly in pathogenic organisms (e.g. using fluorescence-based, or phenotypic assays).

\section{SICLOPPS in eukaryotes}

The majority of reported SICLOPPS screens have been conducted in E. coli, which is an ideal host due to its high transformation efficiency, relative ease of handling and rapid growth time. But there has also been a handful of reports using these libraries in eukaryotic cell-based assays. In one example, SICLOPPS was used in a Saccharomyces cerevisiae (yeast) screen for the identification of cyclic peptides that reduced the toxicity of $\alpha$-synuclein, a key protein in Parkinson's disease [18]. This 
phenotype screen identified two cyclic peptides that specifically reduced the toxicity of human $\alpha$ synuclein, and prevented dopaminergic neuron loss in a Caenorhabditis elegans Parkinson's model. It should be noted that one limitation with screening in yeast is the relatively low transformation efficiency compared to E. coli. In the above work for example, the number of transformants (5 million) was lower than the number of individual cyclic peptides in the library (1.28 billion).

In another example, a modified SICLOPPS library with a mutant (N36A) C-terminal intein that does not splice beyond the lariat intermediate (Figure 1), was combined with a yeast two-hybrid screen to identify cyclic peptides that bind the bacterial SOS response protein LexA [34]. This system was also used for the identification of cyclic peptides that bind and inhibit Abl kinase. Interestingly, the most potent inhibitor developed was a 12-mer cyclic peptide that strongly bound Abl kinase (H396P) with a $K_{\mathrm{d}}$ of $27 \mathrm{nM}$, but this peptide only weakly inhibited the activity of the same protein with an $\mathrm{IC}_{50}$ of $111.3 \mu \mathrm{M}$ [35], illustrating the dichotomy between high affinity binding and potent inhibition of function when using affinity-based screens to identify inhibitors. Nonetheless, this work demonstrates the identification of high affinity peptides from SICLOPPS libraries, however it is unclear whether this is due to the larger ring sizes used (12-mers), or because an affinity-based screen was conducted.

SICLOPPS libraries have also been used in mammalian cells. In a landmark example, a retroviral expression system was adapted to enable cyclic peptide library production in human B cells. The SICLOPPS library was screened for compounds that inhibit interleukin-4 signaling using a functional genetic screen that linked IL-4 inhibition to cell survival and GFP fluorescence [19]. The screen identified several cyclic peptides that inhibited IL-4-mediated germ line epsilon transcription, a potential therapeutic approach for allergy and asthma. More recently, a small SICLOPPS library of 94 cyclic peptides based on segments of a 75 amino acid protein that disrupts the interaction between Aurora B and IN-box was constructed and screened in human cells [36]. The phenotype assay used microscopy to identify abnormal nuclei fragmentation as an indicator of chromosome passenger complex regulation of mitosis. Twenty cyclic peptides were identified that affected this process and were used to illustrate the biological function of the targeted protein-protein interaction in human cells. Another example of using SICLOPPS inteins in human cells is our recently reported approach for delivering our cyclic peptide HIF-1 inhibitor to cells; we engineered a construct that conditionally encodes the $\mathrm{Npu}$ variants of the parent SICLOPPS intein for the production of our HIF-1 inhibitor (cyclo-CLLFVY [26]) onto the chromosome of human HEK-239 cells [20]. The promoter used only produces the inteins in hypoxia and in the presence of doxycycline, therefore allowing the controlled production of our cyclic peptide HIF-1 inhibitor in hypoxic cells incubated with doxycycline. This approach negates the need to chemically synthesize and deliver this cyclic peptide to cell for biological studies, and can be applied to other cyclic peptides.

\section{Outlook}


There is significant current interest around the use of cyclic peptide scaffolds in drug discovery, especially for early stage hit discovery against intractable targets $[37,38]$. There is a growing body of evidence in the literature that supports the drive towards these molecules, which do not necessary fit in with conventional dogma in drug discovery and development $[39,40]$. The SICLOPPS methodology detailed here is one of a handful of techniques available for the generation of macrocycle libraries of hundreds of millions of members, and is arguably one of the simplest to adopt, and most flexible methods for library generation. In addition, the ability to interface SICLOPPS libraries with any cellbased assay and to conduct functional screens (rather than affinity-based assays) is a significant benefit of this technique.

As for how to progress cyclic peptides toward the clinic beyond the initial discovery of a hit, there are two schools of thought. One aims to maintain the cyclic peptide scaffold while improving affinity and cell permeability through the incorporation of non-natural amino acids and backbone modifications, while the other aims to derive more drug-like entities from the initial cyclic peptide hit. The latter may be achieved by identifying the active motif of the cyclic peptide through structural studies or by alanine scanning, and that information used to derive or design more drug-like small molecule inhibitors. We have favored this approach, having demonstrated the conversion of a SICLOPPS derived hexameric cyclic peptide to a more potent ( $K_{\mathrm{d}}$ of $17 \mu \mathrm{M}$ to $685 \mathrm{nM}$ ) small molecule [41], that is active in vivo [42] (Figure 2).

Beyond their use in drug discovery, SICLOPPS cyclic peptides have the potential to serve as rapidly derived chemical tools to inform biological studies or to validate the relevance of a given protein-protein interaction prior to larger scale screening efforts. Studies that solely employ gene knockout or knockdown (e.g. siRNA) remove the targeted protein from the cell rather than inhibit a given proteinprotein interaction or its function. A cyclic peptide inhibitor has the potential to serve alongside these existing biological methods, and help improve target validation steps. 

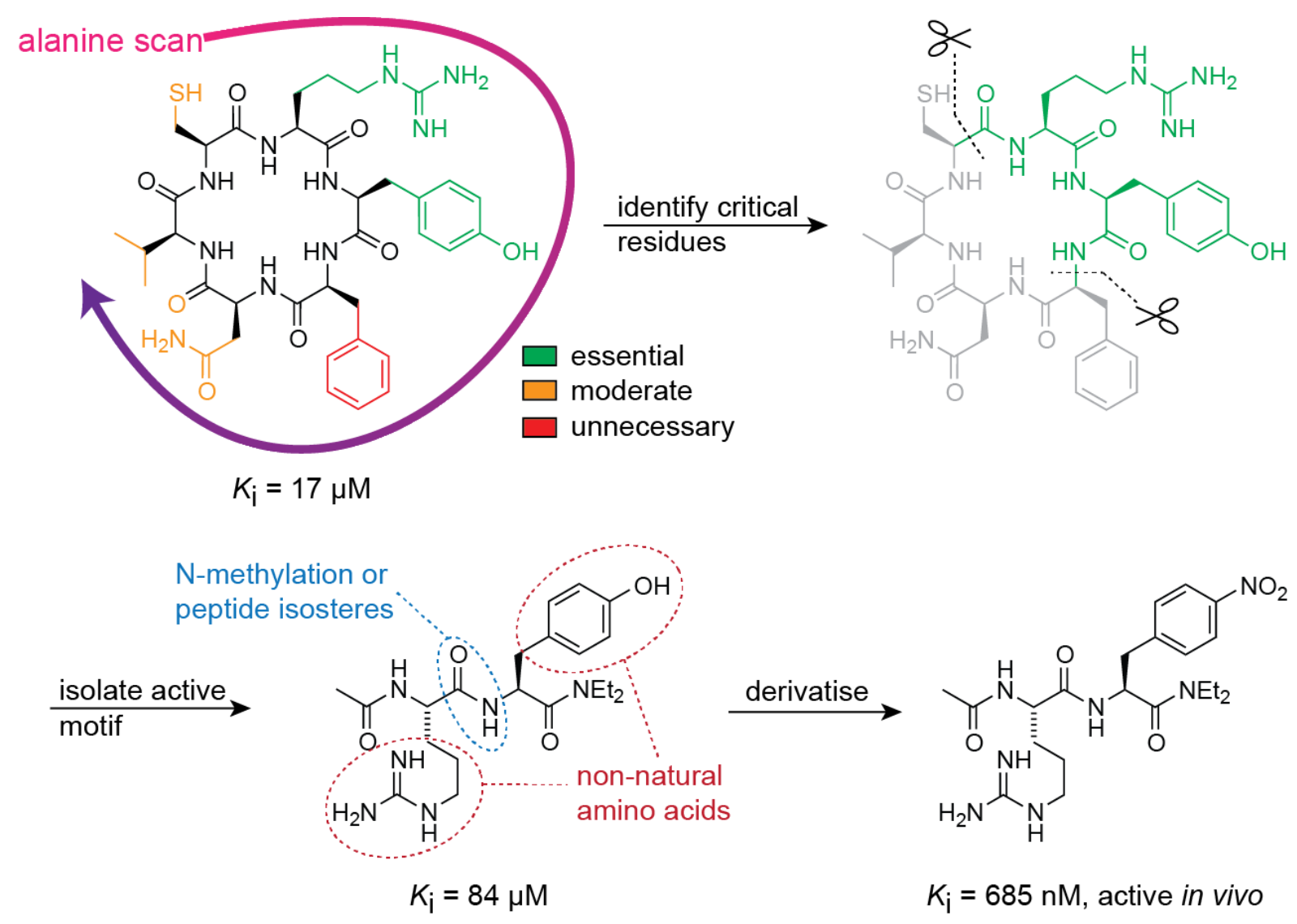

Figure 2. Deriving small molecules from SICLOPPS hits. The initial cyclic peptide, cyclo-CRYFNV, was identified as an inhibitor of ATIC homodimerization (which has an interaction interface of $\sim 5000 \AA^{2}$ ) with a $K_{\mathrm{i}}$ of $17 \mu \mathrm{M}$ [17]. The residues critical for activity were identified by alanine scanning, and a new molecule designed based on this information. While the resulting molecule had lost $\sim 5$-fold activity, this was attributed to the increased conformational flexibility arising from elimination of the cyclic peptide backbone. A series of analogues were synthesized that incorporated non-natural amino acids and N-methylation of the backbone to give a cell permeable small molecule ATIC inhibitor with a $K_{\mathrm{i}}$ of $685 \mathrm{nM}$ [41] that was demonstrated be active in vivo [42].

\section{References}

1. Chen S, Rentero Rebollo I, Buth SA, Morales-Sanfrutos J, Touati J, Leiman PG, Heinis C: Bicyclic peptide ligands pulled out of cysteine-rich peptide libraries. J Am Chem Soc 2013, 135:6562-6569.

- 2. Josephson K, Ricardo A, Szostak JW: mRNA display: from basic principles to macrocycle drug discovery. Drug Discov Today 2014, 19:388-399.

This review is an excellent and detailed discussion of the various mRNA-diplay methods and their use in drug discovery.

3. Kawakami T, Ohta A, Ohuchi M, Ashigai H, Murakami H, Suga H: Diverse backbone-cyclized peptides via codon reprogramming. Nat Chem Biol 2009, 5:888-890.

4. Passioura $\mathrm{T}$, Suga $\mathrm{H}$ : Flexizyme-mediated genetic reprogramming as a tool for noncanonical peptide synthesis and drug discovery. Chemistry 2013, 19:6530-6536.

5. Smith JM, Frost JR, Fasan R: Emerging strategies to access peptide macrocycles from genetically encoded polypeptides. J Org Chem 2013, 78:3525-3531. 
- 6. Passioura T, Katoh T, Goto Y, Suga H: Selection-Based Discovery of Druglike Macrocyclic Peptides. Annu Rev Biochem 2014.

This review is an excellent and very comprehensive look at the various stratergies employed for the discovery of macrocyclic peptide inhibitors.

7. Marsault E, Peterson ML: Macrocycles are great cycles: applications, opportunities, and challenges of synthetic macrocycles in drug discovery. J Med Chem 2011, 54:1961-2004.

8. White CJ, Yudin AK: Contemporary strategies for peptide macrocyclization. Nat Chem 2011, 3:509-524.

9. Perler FB: InBase, the Intein Database. Nucleic Acids Res 2000, 28:344-345.

10. Scott CP, Abel-Santos E, Wall M, Wahnon DC, Benkovic SJ: Production of cyclic peptides and proteins in vivo. Proc Natl Acad Sci U S A 1999, 96:13638-13643.

11. Wu H, Hu Z, Liu XQ: Protein trans-splicing by a split intein encoded in a split DnaE gene of Synechocystis sp. PCC6803. Proc Natl Acad Sci U S A 1998, 95:9226-9231.

- 12. Townend JE, Tavassoli A: Traceless Production of Cyclic Peptide Libraries in E. coli. ACS Chem Biol 2016, 11:1624-1630.

This paper is important as it reports next generation SICLOPPS inteins that use fast splicing Npu inteins and illustrates their benefits over the classically used Ssp inteins.

- 13. Osher EL, Tavassoli A: Intracellular Production of Cyclic Peptide Libraries with SICLOPPS. Methods Mol Biol 2017, 1495:27-39.

This paper is useful as it details the steps necessary for the construction of a SICLOPPS plasmid library.

14. Tavassoli A, Benkovic SJ: Split-intein mediated circular ligation used in the synthesis of cyclic peptide libraries in E. coli. Nat Protoc 2007, 2:1126-1133.

15. Lennard KR, Tavassoli A: Peptides come round: using SICLOPPS libraries for early stage drug discovery. Chemistry 2014, 20:10608-10614.

16. Horswill AR, Savinov SN, Benkovic SJ: A systematic method for identifying small-molecule modulators of protein-protein interactions. Proc Natl Acad Sci U S A 2004, 101:1559115596.

17. Tavassoli A, Benkovic SJ: Genetically selected cyclic-peptide inhibitors of AICAR transformylase homodimerization. Angew Chem Int Ed Engl 2005, 44:2760-2763.

18. Kritzer JA, Hamamichi S, McCaffery JM, Santagata S, Naumann TA, Caldwell KA, Caldwell GA, Lindquist S: Rapid selection of cyclic peptides that reduce alpha-synuclein toxicity in yeast and animal models. Nat Chem Biol 2009, 5:655-663.

19. Kinsella TM, Ohashi CT, Harder AG, Yam GC, Li W, Peelle B, Pali ES, Bennett MK, Molineaux SM, Anderson DA, et al.: Retrovirally delivered random cyclic Peptide libraries yield inhibitors of interleukin-4 signaling in human B cells. J Biol Chem 2002, 277:37512-37518.

- 20. Mistry IN, Tavassoli A: Reprogramming the Transcriptional Response to Hypoxia with a Chromosomally Encoded Cyclic Peptide HIF-1 Inhibitor. ACS Synth Biol 2017. DOI: 10.1021/acssynbio.6b00219

This paper is important as it describes the incorporation of SICLOPPS inteins onto the chromosome of human cells, and represents a new approach to the dlivery of cyclic peptides to human cells.

21. Young TS, Young DD, Ahmad I, Louis JM, Benkovic SJ, Schultz PG: Evolution of cyclic peptide protease inhibitors. Proc Natl Acad Sci U S A 2011, 108:11052-11056. 
22. Kawakami T, Ishizawa T, Fujino T, Reid PC, Suga H, Murakami H: In vitro selection of multiple libraries created by genetic code reprogramming to discover macrocyclic peptides that antagonize VEGFR2 activity in living cells. ACS Chem Biol 2013, 8:1205-1214.

23. Schlippe YV, Hartman MC, Josephson K, Szostak JW: In vitro selection of highly modified cyclic peptides that act as tight binding inhibitors. J Am Chem Soc 2012, 134:10469-10477.

24. Iwai H, Zuger S, Jin J, Tam PH: Highly efficient protein trans-splicing by a naturally split DnaE intein from Nostoc punctiforme. FEBS Lett 2006, 580:1853-1858.

25. Birts CN, Nijjar SK, Mardle CA, Hoakwie F, Duriez PJ, Blaydes JP, Tavassoli A: A cyclic peptide inhibitor of C-terminal binding protein dimerization links metabolism with mitotic fidelity in breast cancer cells. Chemical Science 2013, 4:3046-3057.

-• 26. Miranda E, Nordgren IK, Male AL, Lawrence CE, Hoakwie F, Cuda F, Court W, Fox KR, Townsend PA, Packham GK, et al.: A cyclic peptide inhibitor of HIF-1 heterodimerization that inhibits hypoxia signaling in cancer cells. J Am Chem Soc 2013, 135:10418-10425.

This paper is significant as it illustrates to potential for SICLOPPS screens; it details the discovery and characterization of the first isoform specific HIF-1 inhibitor from a SICLOPPS cyclic peptide library.

27. Tavassoli A, Lu Q, Gam J, Pan H, Benkovic SJ, Cohen SN: Inhibition of HIV budding by a genetically selected cyclic peptide targeting the Gag-TSG101 interaction. ACS Chem Biol 2008, 3:757-764.

28. Datta S, Bucks ME, Koley D, Lim PX, Savinov SN: Functional profiling of p53-binding sites in Hdm2 and Hdmx using a genetic selection system. Bioorg Med Chem 2010, 18:6099-6108.

29. Kjelstrup S, Hansen PM, Thomsen LE, Hansen PR, Lobner-Olesen A: Cyclic peptide inhibitors of the beta-sliding clamp in Staphylococcus aureus. PLoS One 2013, 8:e72273.

30. Naumann TA, Tavassoli A, Benkovic SJ: Genetic selection of cyclic peptide Dam methyltransferase inhibitors. Chembiochem 2008, 9:194-197.

31. Cheng L, Naumann TA, Horswill AR, Hong SJ, Venters BJ, Tomsho JW, Benkovic SJ, Keiler KC: Discovery of antibacterial cyclic peptides that inhibit the ClpXP protease. Protein Sci 2007, 16:1535-1542.

32. El-Mowafi SA, Alumasa JN, Ades SE, Keiler KC: Cell-based assay to identify inhibitors of the HfqsRNA regulatory pathway. Antimicrob Agents Chemother 2014, 58:5500-5509.

33. El-Mowafi SA, Sineva E, Alumasa JN, Nicoloff H, Tomsho JW, Ades SE, Keiler KC: Identification of inhibitors of a bacterial sigma factor using a new high-throughput screening assay. Antimicrob Agents Chemother 2015, 59:193-205.

34. Barreto K, Bharathikumar VM, Ricardo A, DeCoteau JF, Luo Y, Geyer CR: A genetic screen for isolating "lariat" Peptide inhibitors of protein function. Chem Biol 2009, 16:1148-1157.

35. Bharathikumar VM, Barreto K, Decoteau JF, Geyer CR: Allosteric lariat Peptide inhibitors of abl kinase. Chembiochem 2013, 14:2119-2125.

36. Gohard FH, St-Cyr DJ, Tyers M, Earnshaw WC: Targeting the INCENP IN-box-Aurora B interaction to inhibit CPC activity in vivo. Open Biol 2014, 4:140163.

37. Thapa P, Zhang RY, Menon V, Bingham JP: Native chemical ligation: a boon to peptide chemistry. Molecules 2014, 19:14461-14483.

38. Fosgerau K, Hoffmann T: Peptide therapeutics: current status and future directions. Drug Discov Today 2015, 20:122-128.

39. White AM, Craik DJ: Discovery and optimization of peptide macrocycles. Expert Opin Drug Discov 2016, 11:1151-1163.

40. Doak BC, Kihlberg J: Drug discovery beyond the rule of 5 - Opportunities and challenges. Expert Opin Drug Discov 2017, 12:115-119. 
41. Spurr IB, Birts CN, Cuda F, Benkovic SJ, Blaydes JP, Tavassoli A: Targeting tumour proliferation with a small-molecule inhibitor of AICAR transformylase homodimerization. Chembiochem 2012, 13:1628-1634.

- 42. Asby DJ, Cuda F, Beyaert M, Houghton FD, Cagampang FR, Tavassoli A: AMPK Activation via Modulation of De Novo Purine Biosynthesis with an Inhibitor of ATIC Homodimerization. Chem Biol 2015, 22:838-848.

This paper is significant as it illustrates the viability of deriving small molecule inhibitors (that are functional in vivo) from SICLOPPS hits. 